\title{
Preface to "Environmental footprints induced by geo-energy exploitation: the shale gas case"
}

\author{
Paolo Capuano ${ }^{1}$ \\ Published online: 9 February 2019 \\ (c) Institute of Geophysics, Polish Academy of Sciences \& Polish Academy of Sciences 2019
}

In the middle of the first decade of this century, new technological innovations enabled the extraction of natural gas through the use of hydraulic fracturing within gas-bearing shale and other unconventional energy reserves. As a consequence, many places, often in economically lagging rural areas, saw dramatic change as they were socially and economically transformed through rapid natural resource development. The rapid growth of unconventional oil and gas in recent years, particularly from shale formations, involves infrastructure development (well pads, roads and pipelines), well drilling (directional drilling) and stimulation (hydraulic fracturing), and production; all of which has caused concern having the potential to affect the surrounding environment, human health and public safety.

Specifically, the increase in shale gas extraction, attributable to technological advancements which have made natural gas extraction from low permeability shale formations feasible, has generated some concerns about environmental impacts such as the migration of natural gas into water resources and atmosphere and the generation of induced seismicity. Because of this rapid growth, there have been many efforts to assess potential negative impacts in order to find ways to mitigate them and increase the safety of the natural gas industry.

To address specifically one major concern of shale gas exploration and extraction: the associated environmental risks, the European Union launched in 2014 an H2020 call: "Understanding, preventing and mitigating the potential environmental impacts and risks of Shale Gas Exploration and Exploitation" (work programme topic LCE-16-2014). This program funded four projects including SHEER (Shale Gas Exploration and Exploitation Induced Risks) whose objective was to develop best practices for assessing and

Paolo Capuano

pcapuano@unisa.it

1 Department of Physics "E.R Caianiello", University of Salerno, Fisciano, Italy mitigating the environmental footprint of shale gas exploration and exploitation. The consortium includes partners from Italy, UK, Poland, Germany, the Netherlands and USA.

SHEER has developed a probabilistic procedure for assessing short- and long-term risks associated with groundwater contamination, air pollution and induced seismicity. The severity of each of them depends strongly on the unexpected enhanced permeability pattern, which may develop as an unwanted by-product of the fracking processes and may become pathway for gas and fluid migration toward underground water reservoirs or the surface. An important part of SHEER has been devoted to monitor and understand how far this enhanced permeability pattern can be developed both in space and time. These hazards may be at least partially inter-related as they all depend on this enhanced permeability pattern. Therefore, they have been approached from a multi-hazard, multi-parameter perspective. SHEER has developed methodologies and procedures to track and model fracture evolution around shale gas exploitation sites and a robust statistically based, multi-parameter methodology to assess environmental impacts and risks across the operational lifecycle of shale gas.

The developed methodologies have been applied and tested on a comprehensive database (SHEERWER) consisting of seismicity, changes of the quality of groundwaters and air, and operational data (i.e., data important for hazard assessment), collected from past case studies. To preserve and curate the data for long-term use, after the project completion, most of the SHEER database was transferred to the IS-EPOS (The European Observing System, a research infrastructure for Solid Earth science) e-platform and hosted on EPOS TCS AH (EPOS Thematic Core Service Anthropogenic Hazards), by which means the data are made openly accessed and available to the research community. Access to and sharing of the collected data were recognized by Project Consortium as essential for advancement of science.

The database from past case studies has been enriched by the high-quality data collected by SHEER in a planned 
hydraulic fracturing site carried out by the Polish Oil and Gas Company in Wysin (Pomerania, Poland). The plan for this aim has included continuous monitoring groundwater quality, air conditions and seismicity before, during and after the hydrofracturing. In fact, one of the main challenges of SHEER was the implementation of a dedicated multidisciplinary monitoring system, in order to jointly assess, for the first time in Europe, the short- and long-term environmental impacts of shale gas exploration and exploitation operations. The objectives of the monitoring activity were:

- to establish background levels of seismicity, water condition and air pollution,

- to sample the actual impact of fracking on the seismicity, water and air conditions in the direct vicinity and during fracking operations,

- to recognize protracted effects of shale gas operations on the environment,

- to provide observational data to be analyzed in the project.

The detection and characterization of induced seismicity, its role to image fracture processes and fluid transfers and the assessment of seismic hazard as key feature for the SHEER project that wished to advance the current state of the art on the assessment of induced seismicity, providing recommendations toward the definition of monitoring and analysis guidelines. A series of recommendations for best practice in hydrogeological monitoring of future shale gas developments has been produced. The assessment of the impact of the exploration of shale gas activities on the ambient air quality in the vicinity of wells situated in the Pomerania site was achieved through a detailed analysis of the data collected at an air quality station located near the pad.

The multi-hazard risk (MHR) framework developed in SHEER aims at providing a quantitative framework for assessing, in probabilistic terms, the likelihood of occurrence of different possible risk pathway scenarios potentially impacting the surrounding environment. The probabilistic framework developed has been conceived to be a flexible tool for the evaluation of a wide variety of incidental scenarios. In general terms, the adopted method relies on a logic structure based in a bow-tie (BT) approach, which implies the combined use of fault trees (FT) and event trees (ET) that are combined with a wide range of probabilistic tools that rely on Bayesian data analysis concepts. The key characteristics of the developed framework are that it allows the analyst to encode information from different typologies of processes, to include different sources of information and to propagate uncertainties.

Finally, SHEER produces guidelines for monitoring and for risk management. In the first case, the guidelines define early observation standards for monitoring the effects of human activities, such as the hydraulic fracturing techniques on environment and establish monitoring procedures and protocols, considering methods to analyze the effects of induced seismicity, groundwater contamination and air pollution. In the second case, the aims were to understand risk management in the context of shale gas exploitation and to develop a framework for stakeholders and policy makers within which to assess and manage risk in future developments. The leading international standard on risk management is ISO 31000:2018 which was used to critically analyze the risk assessment process and generate a new framework within which to assess risks in shale gas developments, through an approach based on a PESTLE (Political, Economic, Social, Technological, Legal, Environmental) analysis. Much care has been devoted into maintaining and increasing project awareness, research dissemination, external communication and stakeholder engagement.

The best practices to be applied in Europe to monitor and minimize any environmental impacts have been worked with the involvement of an advisory group including governmental decisional bodies and private industries.

The novel scientific concepts and current practices and recommendation for assessing and mitigating the environmental footprint of shale gas exploration and exploitation described in this Special Issue show the increasing potential of the monitoring of the activities that can generate environmental impacts for serving the needs of risk management, decision making and the public.

Finally, I would like to thank all the people participating to the SHEER project, especially the young researchers worldwide, have developed their own talent and have achieved their ambitious and innovative scientific goals through this project.This volume is dedicated to the memory of prof. Paolo Gasparini (former coordinator) and prof. Paul Younger that unfortunately passed away during the SHEER project. 